\title{
Pulsed Electromagnetic Field as a Non-Invasive Alternative or Complementary Treatment in $\beta$-Thalassemia
}

\author{
Shahram Eslami ${ }^{1}$, Mohammad Ali Ebrahimzadeh ${ }^{1}$, Obeid M Malekshah ${ }^{2}$, and Hamidreza Mohammadi ${ }^{* 1,3}$ \\ ${ }^{1}$ Pharmaceutical Sciences Research Center, Faculty of Pharmacy, Mazandaran University of Medical Sciences, Sari, Iran \\ ${ }^{2}$ Department of Pharmaceutics, Rutgers, The State University of New Jersey, Piscataway, NJ 08854, USA \\ ${ }^{3}$ Department of Toxicology and Pharmacology, Faculty of Pharmacy, Mazandaran University of Medical Sciences, Sari, Iran
}

*Corresponding author: Hamidreza Mohammadi, Department of Toxicology and Pharmacology, Faculty of Pharmacy, Iran

\section{ARTICLE INFO}

Received: 陆 December 17, 2019

Published: January 07, 2020

Citation: Shahram Eslami, Mohammad Ali Ebrahimzadeh, Obeid M Malekshah, Hamidreza Mohammadi. Pulsed Electromagnetic Field as a Non-Invasive Alternative or Complementary Treatment in $\beta$-Thalassemia. Biomed J Sci \& Tech Res 24(2)-2020. BJSTR. MS.ID.004034.

Keywords: $\beta$-Thalassemia; Pulsed Electromagnetic Field; Noninvasive Treatment

\begin{abstract}
Today, the usual treatment for patients with $\beta$-thalassemia is regular blood transfusions and iron chelating therapy. However, iron chelation may be related to undesirable outcomes. Recently, two main therapeutic methods have been performed; the gene therapy and the induction of fetal hemoglobin ( $\mathrm{HbF}$ ). However, de novo production of clinically applicable levels of adult $\mathrm{Hb}(\mathrm{HbA})$ is difficult. In consideration of the above, new approaches aiming the treatment of patients with severe $\beta$-thalassemia are greatly needed. Due to the fact that transduction with transcription factors such as KLF1 and BCL11A-XL can stimulate $\beta$-globin expression and based on the ability of pulsed electromagnetic field (PEMF) to stimulate specific genes transcription and overexpression, we hypothesize that PEMF exposure can activate cell stress signaling pathways that result in $\beta$-globin expression which is a cell stress response gene. This considered to be a noninvasive, low-cost and non-cytotoxic treatment approach and represents a small, but significant, step towards this goal
\end{abstract}

Abbreviations: HbF: Fetal Hemoglobin; PEMF: Pulsed Electromagnetic Field; KLF1: Krueppel-Like Factor 1; BCL11A: B-Cell Lymphoma/Leukemia 11A; HS: Heat-Shock; SCX: Specific Gene Transcription Scleraxis; VEGF: Vascular Endothelial Growth Factor; DPN: Diabetic Peripheral Neuropathy; HBMSCs: Human Bone Marrow Stromal Cells; Ocn: Osteocalcin; MSCs: Mesenchymal Stem Cells; AD: Alzheimer's Disease; PBMCs: Peripheral Blood Mononuclear Cells; TCs: Tendon Cells

\section{Mini Review}

$\beta$-thalassemia is a monogenic disease that will be caused by $\beta$-globin faulty synthesis and represents a global health problem. According to the $\mathrm{WHO}$, the carrier rate of $\beta$-thalassemia is around $1.5 \%$ of the world population [1]. The usual treatment for patients with $\beta$-thalassemia is based on regular blood transfusions and iron chelation therapy. Despite all of the progress made in this field, transfusion therapy is linked with serious complications and requires iron chelation therapy, which causes undesirable effects and scarce compliance. In comparison to traditional methods, therapeutic approaches such as gene therapy have recently drawn more attention for the treatment of $\beta$-thalassemia [2]. It was shown that by increasing the level of transcription factors such as Krueppel-like factor 1 (KLF1) and B-cell lymphoma/leukemia 11A (BCL11A) in K562 cells, a prominent increase in $\beta$-globin levels with the resultant level equivalent to that of adult cells can be achieved [3]. Experiments in K562 and KU812 cells also proved that stress signals such as heat-shock (HS), UV- and X-irradiation and osmotic shock can increase $\gamma$-globin mRNA and fetal hemoglobin (HbF) levels in differentiating primary human erythroid cells, but only after 3-5 days of treatments [4].

Another stimulant like pulsed electromagnetic field (PEMF), a safer approach compared to other mentioned methods showed 
to be a suitable treatment strategy for inflammatory and tissuespecific gene expression related conditions such as alzheimer, arthritis, bacterial infection and wound healing in diabetic cases and its results demonstrated a significant improvement in the treatment of the patients [5]. The other effects of PEMF are its role in promoting angiogenesis in bone marrow by the overexpression of angiopoietin-2 mRNA [6], and up-regulation of osteogenic factors in human calvarial bone cell cultures which are critical factors in fracture healing $[7,8]$. It also can increase tendon- specific gene transcription scleraxis (SCX) (+95\%) and type I collagen (COL1A1) (+97\%) in human tendon cells culture model [9]. Angiopoietin-2 up-regulation which caused by PEMF treatment can promote endothelial cell migration by loosening their intercellular contacts, and fibroblast growth factor- 2 in the mice bone marrow. These findings suggest that PEMF induces an angiogenesis-prone environment in the bone marrow without having invasive effects including the induction of hypoxic conditions or inflammation [6].

Dose-dependent upregulation in the expression of SCX, and COL1A1 after PEMF treatment of tendon cells (TCs) is particularly important since PEMF stimulates cell proliferation with a consequent positive effect on tendon recovery. The other observation after PEMF treatment is a small, but significant, increase of IL-1 $\beta$ [9]. IL$1 \beta$ induces the production of IL- 6 , a multifunctional Th2 cytokine which exhibits immunoregulatory functions in tissues and plays an essential role in tissue healing, as it is involved in cell proliferation and survival $[9,10]$. Thus, the increase of IL-6 correlates with the amount of cell viability and proliferation observed after PEMF treatment. On the other hand, IL-6 also has a stimulatory effect on IL-10 production [9]. IL-10 is not only the most effective antiinflammatory Th2 cytokine but it also affects connective tissue cells such as fibroblasts and chondrocytes [11]. In tenocytes, upregulation of IL-10 correlates with the healing increase in murine models [12]. The role of IL-10 in tissue repair involves the regeneration of extracellular matrix, especially in elevating elastin [13] and a significant increase in the vascular endothelial growth factor (VEGF). This is in accordance with the observed increase of IL-6, which is the main vascular endothelial growth factor (VEGF) promoter [9].

PEMF promotes angiogenesis in bone marrow, by the overexpression of angiopoietin-2 mRNA [6]. Thus, PEMF positively influences proliferation, tendon-specific marker expression, and the release of anti-inflammatory cytokines and angiogenic factor in a healthy human TCs culture model in a dose-dependent manner [9]. Diabetic peripheral neuropathy (DPN) studies in animal models, demonstrated that the restitution of nerve function induced by PEMF stimulation will lead to down-regulation of VEGF. This down regulation, in turn, causes less damage to peripheral nerve fibers. It is suggested that PEMF might have direct corrective effects in relieving peripheral neuropathic symptoms in diabetic rats with DPN [14]. PEMF stimulation of human bone marrow stromal cells (HBMSCs), in lumbar spinal fusion, affects cell cycle regulation, cell structure, and growth receptors or kinase pathways. In the differentiation and mineralization stages, PEMF regulated preosteoblast gene expression, the growth factor-beta (TGF- $\beta$ ) transformation signaling pathway and microRNA 21 (miR21) activity are highly regulated.

PEMF can affect bone metabolism by activation of the TGF- $\beta$ signaling pathway and stimulation of microRNA 21-5p (miR21$5 p)$ expression in human bone marrow stromal cells (HBMSCs) [15]. In the process of bone lesions repair, PEMF stimulation alone is able to motivate the expression of osteogenic genes that can lead to the higher expression levels of the osteocalcin (Ocn) mRNA in mesenchymal stem cells (MSCs) followed by MSCs proliferation. PEMFs also affect the molecular currents and cause a specific transmembrane signaling which will promote osteogenic differentiation [16]. PEMF is able to modulate both microRNA (miRNAs) that functions in RNA silencing and post-transcriptional regulation of gene expression and mRNA that is involved in the Alzheimer's disease (AD) related pathways, which will lead to the rebalancing of the pathways' deregulation occurring in the AD. In an ex vivo human peripheral blood mononuclear cells (PBMCs) study, a quantitative reduction of $\beta$-secretase, following by PEMF exposure, confirmed the protective role of the electromagnetic field whose action would counteract the formation of $\beta$-amyloid.

Expression values of miR-107 and miR-335-5p that are the negative regulator of enzyme beta-secretase 1 (BACE1) and $\alpha$-amino-3-hydroxy-5-methyl-4-isoxazolepropionic acid (AMPA) receptor, respectively decrease after PEMF exposure, and the same trend can be observed for the expression of miR-26b-5p, which is involved in brain signaling and synaptic plasticity. This possible effect of PEMF exposure confirms the capacity of the electromagnetic field to stimulate both tissue regeneration and brain signaling [17]. According to previous studies, cell stress signals induce $\gamma$-globin gene expression and this induction is a part of physiological stress response in erythroid cells [4]. It was shown that K562 cells and erythroid cells from cord blood progenitors in comparison to the adult cells have absent or lower levels of both KLF1 and BCL11A that are essential in the $\beta$-globin expression. These types of cells also express predominantly (mainly) $\gamma$, with a low level of $\beta$-globin. There are increasing data to show that KLF1 also regulates many other erythroid genes and hence plays a critical and central role in erythropoiesis [3].

Since the current conventional drugs and surgical procedures do not have the ideal combination of efficacy, safety, and ease of use, PEMF therapy can be a good choice for complementary treatment in $\beta$-Thalassemia because it is a noninvasive, low-cost, noncytotoxic treatment which causes significantly lower discomfort compared to conventional therapy and there are few known risks associated with the use of PEMF therapy [5]. Regarding the important role of cell stress in $\gamma$-globin gene induction and based 
on the ability of PEMF to stimulate specific gene transcription and overexpression, we hypothesize that PEMF exposure can activate cell stress signaling pathways that result in $\beta$-globin expression which is a cell stress response gene. For proving this hypothesis, at first the PEMF different dosing parameters has to be studied, such as the intensity, the frequency and the burst width/wait, the pulse width/wait, the pulse rise and the fall time, duration of PEMF application and the distance of magnetic surface from targeted cell or tissue. The obtained information can help to optimize and standardize experimental protocol, to increase KLF1 and BCL11A level, effectively. This increase can elevate the $\beta$-globin expression and finally might be effective in the $\beta$-Thalassemia treatment. Our remark favorites the utility of PEMF as a physical stress to increase $\beta$-globin gene expression in erythroid cells for in vitro and or in vivo experiments in the future.

\section{Conclusion}

The usual treatment for $\beta$-thalassemia that is a combination of blood transfusions and iron chelation therapy has severe side effects that make it necessary for patients to use other medications and procedures like iron chelation therapy. In the pursuit of alternative treatment studies have shown that using heat-shock (HS), UV- and X-irradiation therapy can trigger stress signals that will cause a chain of reaction to activate transcription factors such as KLF1 and BCL11A-XL. These signals can stimulate $\beta$-globin production. Other alternatives like pulsed electromagnetic field (PEMF) might provide a better approach. It was shown in multiple studies that PEMF can cause different effects in patients including healing based on promoting angiogenesis in the bone marrow tissue, immunoregulatory functions based on IL-1 $\beta$, IL-6, and IL-10 production and up-regulation of osteogenic factors and genes like SCX, COL1A.

It also proves to be effective in restitution of nerve function in DPN studies to relieve peripheral neuropathic symptoms and HBMSCs stimulation via TGF- $\beta$ signaling pathway and miR21 activity. Other studies also showed promising therapeutic effects of this treatment on both tissue regeneration and brain signaling. Since all of these effects were reported without having side effects such as the induction of hypoxic conditions or inflammation and based on its noninvasive, low-cost, non-cytotoxic nature, we hypothesize to use it for activating cell stress signaling pathways to initiate and increase $\beta$-globin expression as a cellular stress response gene. This objective will need future studies to evaluate its effectiveness in thalassemic patients and also provide information to optimize and standardize its experimental protocol.

\section{Conflict of Interest}

The authors declare that they have no conflict of interest.

\section{References}

1. Tyan PI, Radwan AH, Eid A, Haddad AG, Wehbe D, et al. (2014) Novel approach to reactive oxygen species in nontransfusion-dependent thalassemia. BioMed research international pp. 350432.
2. Breda L, Rivella S, Zuccato C, Gambari R (2013) Combining gene therapy and fetal hemoglobin induction for treatment of beta-thalassemia. Expert review of hematology 6(3): 255-264.

3. Trakarnsanga K, Wilson MC, Lau W, Singleton BK, Parsons SF, et al. (2014) Induction of adult levels of beta-globin in human erythroid cells that intrinsically express embryonic or fetal globin by transduction with KLF1 and BCL11A-XL. Haematologica 99(11): 1677-1685.

4. Schaeffer EK, West RJ, Conine SJ, Lowrey CH (2014) Multiple physical stresses induce gamma-globin gene expression and fetal hemoglobin production in erythroid cells. Blood cells, molecules \& diseases 52(4): 214-224.

5. Ross CL, Harrison BS (2013) The use of magnetic field for the reduction of inflammation: a review of the history and therapeutic results. Alternative therapies in health and medicine 19(2): 47-54.

6. Goto T, Fujioka M, Ishida M, Kuribayashi M, Ueshima K, et al. (2010) Noninvasive up-regulation of angiopoietin-2 and fibroblast growth factor-2 in bone marrow by pulsed electromagnetic field therapy. Journal of orthopaedic science 15(5): 661-665.

7. Clark CC, Wang W, Brighton CT (2014) Up-regulation of expression of selected genes in human bone cells with specific capacitively coupled electric fields. Journal of orthop Res 32(7): 894-903.

8. Goodwin TJ (2014) Modifying the genetic regulation of bone and cartilage cells and associated tissue by EMF stimulation fields and uses thereof. USA.

9. De Girolamo L, Stanco D, Galliera E, Vigano M, Colombini A, et al. (2013) Low frequency pulsed electromagnetic field affects proliferation, tissuespecific gene expression, and cytokines release of human tendon cells. Cell biochemistry and biophysics 66(3): 697-708.

10. Malekshah OM, Lage H, Bahrami AR, Afshari JT, Behravan J (2012) PXR and NF-kappaB correlate with the inducing effects of IL-1beta and TNFalpha on ABCG2 expression in breast cancer cell lines. European journal of pharmaceutical sciences 47(2): 474-480.

11. Schulze Tanzil G, Zreiqat H, Sabat R, Kohl B, Halder A, et al. (2009) Interleukin-10 and articular cartilage: experimental therapeutical approaches in cartilage disorders. Current gene therapy 9(4): 306-315.

12. Ricchetti ET, Reddy SC, Ansorge HL, Zgonis MH, Van Kleunen JP, et al. (2008) Effect of interleukin-10 overexpression on the properties of healing tendon in a murine patellar tendon model. The Journal of hand surgery 33(10): 1843-1852.

13. John T, Lodka D, Kohl B, Ertel W, Jammrath J, et al. (2010) Effect of proinflammatory and immunoregulatory cytokines on human tenocytes. Journal of orthopaedic research 28(8): 1071-1077.

14. Lei T, Jing D, Xie K, Jiang M, Li F, et al. (2013) Therapeutic effects of 15 $\mathrm{Hz}$ pulsed electromagnetic field on diabetic peripheral neuropathy in streptozotocin-treated rats. PloS one 8(4): e61414.

15. Selvamurugan N, He Z, Rifkin D, Dabovic B, Partridge NC (2017) Pulsed Electromagnetic Field Regulates MicroRNA 21 Expression to Activate TGF-beta Signaling in Human Bone Marrow Stromal Cells to Enhance Osteoblast Differentiation. Stem cells international 2017: 2450327.

16. Jazayeri M, Shokrgozar MA, Haghighipour N, Bolouri B, Mirahmadi F, et al. (2017) Effects of Electromagnetic Stimulation on Gene Expression of Mesenchymal Stem Cells and Repair of Bone Lesions. Cell journal 19(1): 34-44.

17. Capelli E, Torrisi F, Venturini L, Granato M, Fassina L, et al. (2017) LowFrequency Pulsed Electromagnetic Field Is Able to Modulate miRNAs in an Experimental Cell Model of Alzheimer's Disease. Journal of Healthcare Engineering 2017: 2530270. 
ISSN: 2574-1241

DOI: 10.26717/BJSTR.2020.24.004034

Hamidreza Mohammadi. Biomed J Sci \& Tech Res

(c) 9 This work is licensed under Creative Commons Attribution 4.0 License

Submission Link: https://biomedres.us/submit-manuscript.php

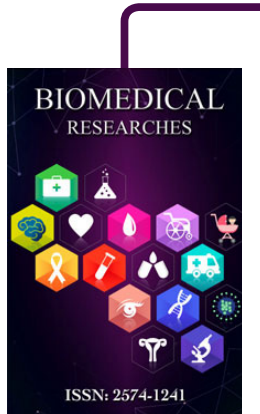

Assets of Publishing with us

- Global archiving of articles

- Immediate, unrestricted online access

- Rigorous Peer Review Process

- Authors Retain Copyrights

- Unique DOI for all articles

https://biomedres.us 